\title{
Confinement and Integration Density of Plasmonic Waveguides
}

\author{
X. Sun, Student Member, IEEE, M. Z. Alam, Member, IEEE, M. Mojahedi, Senior Member, IEEE, \\ and J. S. Aitchison, Senior Member, IEEE
}

\begin{abstract}
In general, plasmonic waveguides exhibit a tradeoff between propagation loss and mode confinement-the smaller the mode size, the higher the propagation loss. We compare the confinement and loss of three commonly used plasmonic waveguides. We define two distinct figures of merit based on application for which the plasmonic waveguide is designed: 1) applications which require high power density and 2) applications which require high lateral packing density. The first figure of merit is based on the power density and propagation loss, whereas the second depends on the coupling length and the propagation loss. We have identified the relative advantages and limitations of the plasmonic waveguides for these different applications.
\end{abstract}

Index Terms-Photonic integrated circuits, plasmonic waveguide, propagation losses, mode confinement.

\section{INTRODUCTION}

I N RECENT years, sub-wavelength confinement of light has attracted much attention due to potential applications in on-chip interconnects and bio-sensing. A dielectric waveguide, with a high refractive index contrast can dramatically increase the mode confinement; however, the mode size achievable in a dielectric waveguide is still limited by diffraction [1]. Another approach to achieving a high mode confinement is based on the use of plasmonics; i.e., the application of surface plasmons polaritons (SPP). Waveguides based on SPP can break the diffraction limit associated with dielectric waveguides and a subwavelength confinement can be achieved. The plasmonic waveguide have become popular because of a combination of features including: high field intensity at the metal interface, resonant behavior, slow group velocity and the ability to confine light on the nanometer scale [1]-[3]. Many different kinds of plasmonic waveguides have been proposed [4]-[7] and many applications have been suggested [8]-[13] to utilize these properties. Unlike dielectric waveguides for which the propagation loss can be negligible, the presence of metal as part of the guiding structure makes plasmonic guides highly lossy; thus limit the propagation length of a plasmonic waveguide [1]. To realize the full potential of plasmonic waveguides, either in sensing, nonlinear

Manuscript received October 1, 2014; revised November 24, 2014; accepted November 26, 2014. This work was supported by the Natural Sciences and Engineering Research Council of Canada CREATE ASPIRE-Applied Science in Photonics and Innovative Research in Engineering.

The authors are with the Department of Electrical and Computer Engineering, University of Toronto, Toronto, ON M5S 3G4, Canada (e-mail: xiaoxiao.sun@utoronto.ca; muhammad.alam@mail.utoronto.ca; mojahedi@ waves.utoronto.ca; stewart.aitchison@utoronto.ca).

Color versions of one or more of the figures in this paper are available online at http://ieeexplore.ieee.org.

Digital Object Identifier 10.1109/JSTQE.2014.2377636
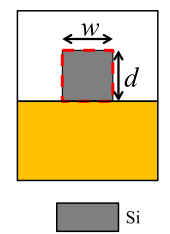

(a)

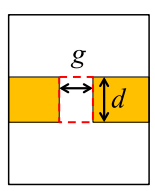

(b)

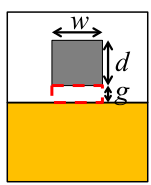

$\mathrm{SiO}_{2}$

(c)
Fig. 1. Schematic depiction of (a) the dielectric loaded surface plasmon waveguide, (b) the plasmonic slot waveguide, (c) the hybrid plasmonic waveguide, and (d) the silicon waveguide.

applications or interconnects, it is important to compare different plasmonic waveguides and identify the best design for a particular application.

The purpose of this work is twofold. The first objective is to investigate the characteristics of some important plasmonic waveguide configurations. Our second objective is to compare different plasmonic waveguides based on the results obtained from our analysis. In particular, we consider the use of plasmonic waveguides for a nonlinear or sensing application where a high optical power density is required and an integration application where multiple waveguides need to be placed in close proximity.

There are many different kinds of plasmonic waveguide which have been proposed in last few years. Here we compare three popular plasmonic waveguides, as shown schematically in Fig. 1(a)-(c): dielectric loaded plasmon waveguide (DLSPW), plasmonic slot waveguide, and hybrid plasmonic waveguide (HPWG). The DLSPW is a typical single interface plasmonic waveguide which offers confinement using the single metal interface, the plasmonic slot waveguide can further squeeze the mode by using two metal interfaces, and the HPWG uses the coupled plasmonic and dielectric mode to confine the light. We intend to compare the relative performance of these guides. In addition we also intend to compare the performance of these waveguides with those of a typical silicon waveguide as shown in Fig. 1(d). The performance of the silicon waveguide will be used as the reference to assess if the plasmonic waveguides really offer some advantages over purely dielectric waveguides.

The paper is organized as follows. In Section II we review the criteria used in our work for the evaluation of the plasmonic guides and explain the rationale for choosing these criteria. In Section III we investigate the guided power density and confinement of the guides shown in Fig. 1. In Section IV we compare the lateral density achievable for these waveguides. Section V summarizes the results presented in Sections III and IV, and Section VI discusses the relative advantages and limitations of these waveguides. 


\section{METHOD OF COMPARISON}

A comparison of the mode confinement ability of different waveguide configurations is often necessary for integrated optics to help decide which structure is best for a particular application. The modes in a waveguide exhibit a decay of field intensity in the cladding and technically have an infinite extent and need criteria to evaluate the mode size. The mode profiles for dielectric waveguides generally have a regular elliptical shape; and their guiding ability can be described conveniently using the concept of effective mode area [14], [15]. Sometimes for elliptical shape modes in a dielectric waveguide, the mode size could be defined as where the intensity has fallen to $1 / \mathrm{e}^{2}$ of the central peak value [16]. Unlike a typical dielectric waveguide the shapes of the modes supported by plasmonic waveguides are usually more complicated, and as a result defining the area used for mode size calculation is more challenging. Because of the shallow penetration depth of the field of the metal, there is a sharp mode profile transition at the metal-dielectric interfaces. The 1/e field magnitude contour is used for mode size in [17] with limited success to specific waveguides. Four types of definitions of mode size have been introduced in [18], and the conclusions reached from using these definitions do not agree with each other [18]. Another often used criterion is the confinement factor [19]. In general power is concentrated in the high index region for dielectric waveguides. Confinement factor for dielectric waveguides therefore, is an indication of the power confinement in high index core for such guides. The definitions of confinement factor have also been used for comparing plasmonic waveguides but limited to comparing modes in the area of the same dimensions [20]. As a result, evaluation of plasmonic guides for various applications can be a challenging task.

One consideration for integrated photonic circuits is the evaluation of the bend loss versus bend radius. Comparison of several plasmonic waveguides based on this criterion has been reported in [21]. For the sake of brevity this topic will not be further discussed here. Another concern is the amount of power coupling between two adjacent waveguides [22], which is used to evaluate the mode lateral extension (or crosstalk) for both dielectric and plasmonic waveguides. For the plasmonic waveguide, the change of structures or dimensions not only alters the crosstalk but also the propagation losses; however, there is no detail comparison in literature based on this.

Here our proposed comparison methods are based on specific applications, i.e., applications where high power density and/or on-chip packing density is concerned. For all the comparisons, we include the effect of the propagation loss since it is one of the major performance limiting factors for plasmonic waveguides.

We should point out that the focus of this work is to discuss the general characteristics of various plasmonic waveguides, which will help a designer to choose the proper waveguide for a particular application. Therefore, we have not discussed any specific device in this work. The details of various device designs can be found in existing literature, for example [23], [24].

\section{A. Criteria Based on Power Density}

In many applications of plamsonics such as nonlinear optics [9], [10] and biosensing [11]-[13] a the high power density is required to maximize the interaction. For such applications, in addition to having a small mode size, it is also important to confine the power in a specific area of the waveguide region. For example, a typical application of the DLSPW would probably require power confinement in the high index core marked by the dotted line in Fig. 1(a). Application of the slot waveguide, on the other hand will most likely require the power to be concentrated in the slot region indicated by the dotted line in Fig. 1(b). A typical application of HPWG would require high power concentration in the region between the silicon and metal indicated by the dotted line in Fig. 1(c). It is unlikely that one would intend to use HPWG for confining light in the high index medium which can be achieved in much simpler manner as shown in Fig. 1(a) and (d). In order to compare the different types of plasmonic guides, instead of defining mode size as in [16]-[18] or figure of merit as in [17], we are more interested in the region where we want the power to be confined. The dotted areas in Fig. 1 show the typical areas where light would require to be confined for various plasmonic guides.

We use two other criteria: the power confinement factor $(\Gamma)$ and the power density $(D)$ in the area of interest. For the power propagating in the waveguide along the $z$-direction, the confinement factor $\Gamma$ is defined as the fraction of power confined in region $i$ [the area of interest marked by the dotted lines in Fig. 1(a)-(d)] according to [19]

$$
\Gamma=\frac{\iint_{i}\left|<S_{z}(x, y)>\right| d x d y}{\int_{-\infty}^{\infty} \int_{-\infty}^{\infty}\left|<S_{z}(x, y)>\right| d x d y}
$$

Here, $S_{z}$ is the time-averaged Poynting vector along the $z$ direction.

The confinement factor alone is not enough to evaluate the usefulness of particular waveguide geometry. In addition to having a large $\Gamma$ it is also important to have high power density $D$ in the area of interest. To evaluate this we define the power density $D$ in the following manner:

$$
D=\frac{\Gamma \cdot P_{t o t a l}}{A_{i}} .
$$

Here, $A_{i}$ is the area enclosed by the dotted lines in Fig. 1(a)(d) and $P_{\text {total }}$ is the total guided power, which is normalized to $1 \mathrm{mw}$ in the present case.

\section{B. Criteria Based on Lateral Confinement}

Another area where plasmonic waveguides have been proposed is that of on-chip communication systems which are compatible with silicon CMOS. Achieving a high device density without causing significant crosstalk is important for such applications. A good indication of lateral packing density is based on the coupling length $\left(L_{c}\right)$, i.e., the distance over which complete power transfer happens for adjacent waveguides. $L_{c}$ is given by the following expression:

$$
L_{c}=\frac{\lambda}{2\left|n_{e}-n_{o}\right|} .
$$

Here, $n_{e}$ is the refractive index for the even mode and $n_{0}$ is the refractive index for the odd mode. The larger the coupling length, the lower is the crosstalk between guides and the higher 


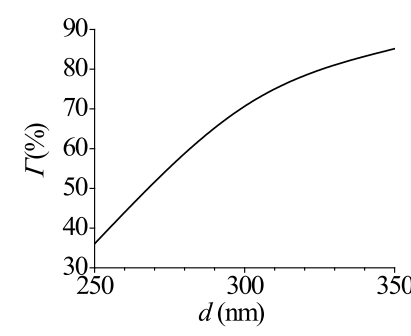

(a)

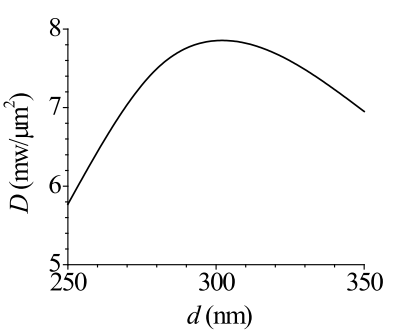

(b)
Fig. 2. Variation of (a) confinement factor and (b) power density with dimensions for the silicon waveguide.

is the achievable packing density for a given level of crosstalk. In this work we will compare the packing density achievable for various plasmonic guides in terms of their coupling lengths.

In the next two sections we use the criterion defined above to evaluate the performance of the waveguides shown in Fig. 1. We use commercial finite difference software Lumerical Mode Solutions to analyze the effects of waveguide dimensions on the performance of the guide. The wavelength of operation is $1.55 \mu \mathrm{m}$ and properties of silver and silicon are taken from [25] and [26] respectively.

\section{CONFInEMENT AND POWER DENSITY OF VARIOUS WAVEGUIDES}

\section{A. Silicon Waveguide}

Fig. 2 shows the effect of waveguide dimension on the confinement factor and power density for a silicon waveguide with a square cross section. As shown in Fig. 2(a), as the size of the silicon core becomes larger; more power is confined in the core. The power density in the core is a maximum for an optimum choice of waveguide dimensions, as shown in Fig. 2(b). Silicon waveguide can confine up to $80 \%$ power in the core. Power density $(D)$ corresponding to this confinement is approximately $7 \mathrm{mw} / \mu \mathrm{m}^{2}$. The highest power density $\left(D=8 \mathrm{mw} / \mu \mathrm{m}^{2}\right)$ is achieved for $d=300 \mathrm{~nm}$, and the corresponding confinement is $70 \%$. As we will see in later sections, the power density achievable for silicon waveguides is much lower than that achievable for plasmonic guides.

As we talk about the confinement ability of a dielectric waveguide, one popular structure that can provide strong confinement in the low index region is the silicon slot waveguide. The optical power inside the slot and corresponding average optical intensity (similar to the power density in this paper) have been carefully analyzed in [27]. For the slot waveguide of dimensions $50 \mathrm{~nm} \times 300 \mathrm{~nm}$, the power density inside the slot is $20 \mathrm{mw} / \mu \mathrm{m}^{2}$ [27]. It will be shown in the later sections that the power density in the low index regions of the HPWG and the plasmonic slot waveguide has been increased because of the existence of metal, although at the cost of increased propagation losses.

\section{B. Dielectric Loaded Surface Plasmon Waveguide}

The DLSPW consists of a high index material on plasmonic metal. The power is confined to the high index core close to metal surface. Although the core can be made of a variety of

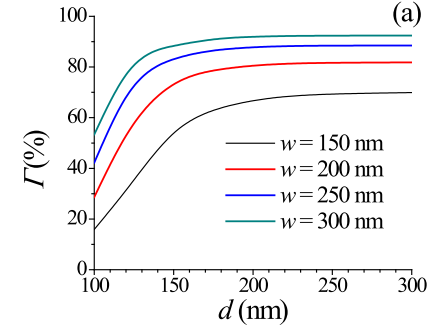

(a)

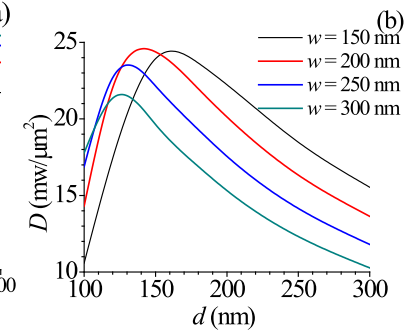

(b)

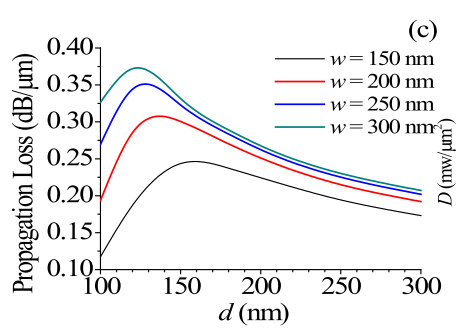

(c)

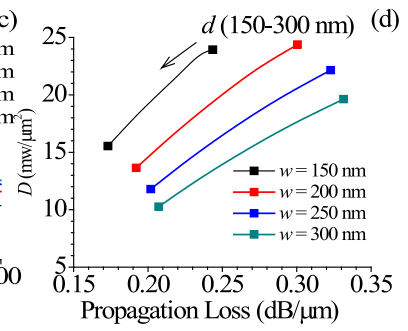

(d)
Fig. 3. Variation of (a) confinement factor, (b) power density, and (c) propagation loss as a function of $d$, and (d) power density versus propagation loss for different $w$ for the DLSPW.

materials, e.g., silicon, polymer, or silica, the highest confinement is achieved when the core is a high index material like silicon. Therefore, in this work we chose to examine the DLSPW consisting of a silicon slab on a silver surface [4]. Fig. 3(a) and (b) show the confinement and power density achievable for the DLSPW. The confinement is comparable to silicon waveguides. For example for a DLSPW with dimensions of $w=200$ $\mathrm{nm}, d=150 \mathrm{~nm}$, about $75 \%$ power is confined in the core, as shown in Fig. 3(a). The corresponding cross section is smaller than that of a silicon waveguide for similar confinement and as a result, the power density is higher $\left(D=25 \mathrm{mw} / \mu \mathrm{m}^{2}\right)$, as shown in Fig. 3(b). For a very small waveguide dimensions the confinement factor and the power density is low because most of the power leaks out to low-index cladding. The power then becomes more confined in silicon for larger dimensions. When $d$ continue increases, the power density drops again due to a larger core. Fig. 3(c) shows the propagation loss. The relation between the propagation loss and the power density are plotted in Fig. 3(d).

\section{Plasmonic Slot Waveguide}

The Plasmonic slot waveguide consists of two finite height metal films separated by a small gap. Since the field cannot penetrate into the metal, the mode is highly confined in the gap between two metal walls. As shown is Fig. 4(a), even for a narrow slot, the power is highly confined in the slot region. For a slot as small as $150 \mathrm{~nm} \times 30 \mathrm{~nm}$, approximately $65 \%$ power is confined in the slot region. The corresponding power densities are shown in Fig. 4(b). The high power confinement in a small gap area results in a very high power density. However, as shown in Fig. 4(c), this high power density is achieved at the price of a very large propagation loss. In order to show the relation of $D$ and loss, we plot the relation of power density with associated loss for different metal height in Fig. 4(d). 


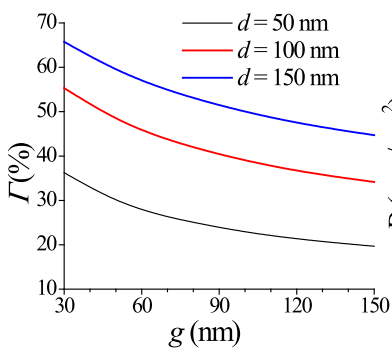

(a)

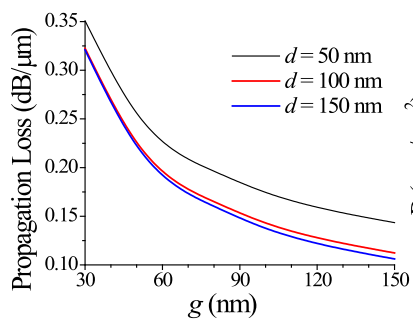

(c)

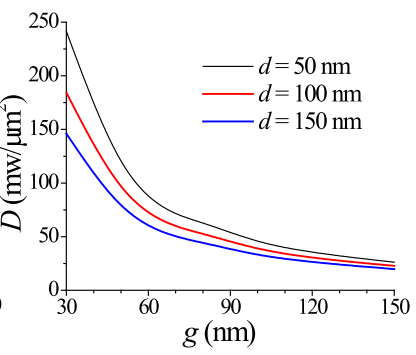

(b)

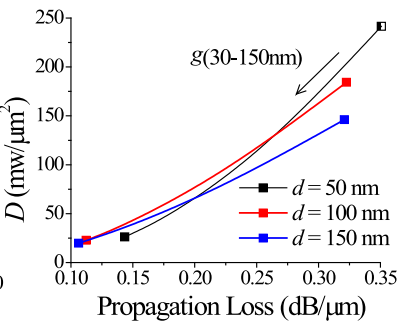

(d)

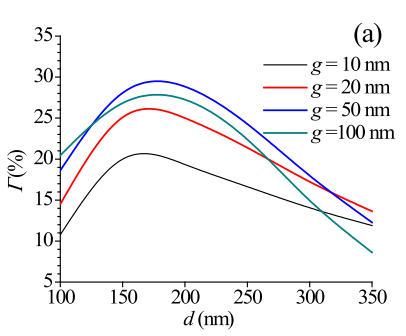

(a)

(c)

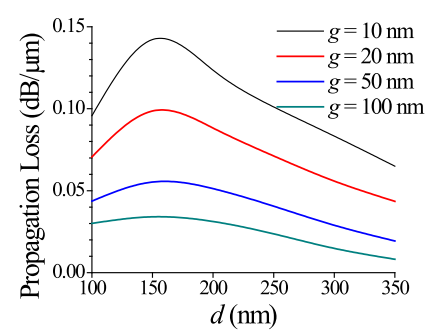

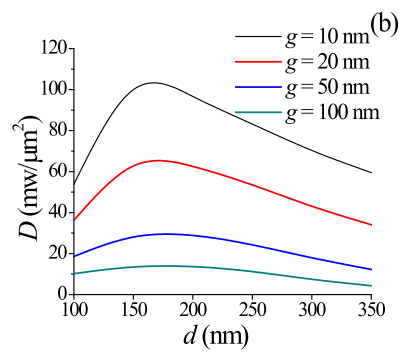

(b)

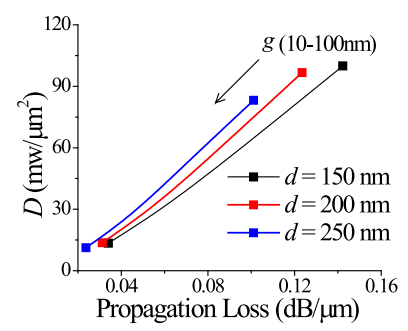

(d)
Fig. 4. Variation of (a) confinement factor, (b) power density, and (c) propagation loss as a function of $g$, and (d) power density versus propagation loss for different $d$ for the plasmonic slot waveguide.

\section{Hybrid Plasmonic Waveguide}

The mode supported by the HPWG is the result of coupling between surface plasmon and dielectric waveguide mode. Power in this guide is confined in the low index region (spacer) between the metal and high index medium. The mode properties are affected by waveguide width $(w)$, thickness of spacer region $(g)$ and thickness of high index region $(d)$. In this work we have fixed waveguide width to $200 \mathrm{~nm}$ since for this width, the HPWG provides good confinement, higher power density and relatively lower propagation distance. We have varied the other two dimensions ( $d$ and $g$ ) and investigated their effects on modal characteristics.

For a thin silicon layer (small $d$ ), the waveguide behave like a pure plasmonic waveguide and most of the power spreads out to the upper cladding layer, thus the power inside the spacer is low, as shown in Fig. 5(a). As the height of the waveguide $d$ increases, the mode becomes more hybrid in nature and the waveguide begins to confine more power inside the spacer region. The highest value is reached when $d=150-200 \mathrm{~nm}$. Further increase $d$ causes the electric field to move into the silicon and the waveguide becomes similar to a dielectric waveguide. This results in a reduction in confinement factor in the spacer. At the same time, increasing the gap from a very small dimension $(g=10 \mathrm{~nm})$ increases the power inside the gap, until the gap becomes too large and confinement is lost. Because the area of gap region is different, smaller gap as $g=10 \mathrm{~nm}$ has the highest power density and highest propagation loss, as shown in Fig. 5(b) and (c). The relation of power density versus propagation loss for hybrid mode $(g=10-100 \mathrm{~nm})$ for fixed $d$ is shown in Fig. 5(d).

\section{Coupling Lengths of VARious WaVEguides}

As mentioned in Section II, we have chosen coupling length as the criterion for evaluating the density of integration achiev-
Fig. 5. Variation of (a) confinement factor, (b) power density, and (c) propagation loss as a function of $d$ for different $g$, and (d) power density versus propagation loss for different $d$ for the HPWG.

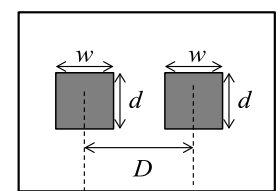

(a)

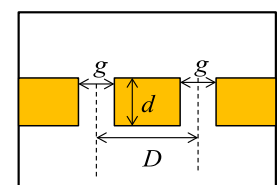

(d)

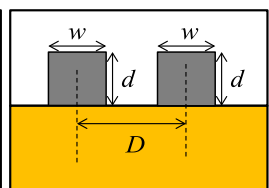

(b)

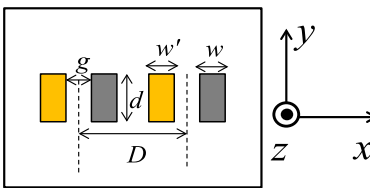

(e)
Fig. 6. Structures analyzed in this work for estimation of crosstalk. (a) Silicon waveguide. (b) Dielectric loaded surface plasmon waveguide. (c) Hybrid plasmonic waveguide. (d) Plasmonic slot waveguide. (e) $90^{\circ}$ rotated HPWG.

able for various waveguides. We compare the coupling length for identical waveguides separated from each other by the same distance $(D)$ as shown in Fig. 6. We have considered two different configurations of the HPWG (see Fig. 6(c) and (e)). The reason for this will be explained later in section.

Two supermodes (even and odd) are supported by two closely spaced identical waveguides. The propagation loss of these two modes is slightly different but quite close. Similar to [28] we define the propagation loss as the average of that of the even and odd modes.

\section{A. Silicon Waveguide}

The coupling length of the silicon waveguide with different center-to-center separation $D$ has been analyzed in [24]. Here we present a similar analysis for the silicon waveguide as a reference. Fig. 7(a) shows the coupling lengths of two rectangular silicon waveguides separated by $1 \mu \mathrm{m}$ center-to-center spacing for various waveguide dimensions. Fig. 7(b) shows the coupling lengths for the same waveguide dimensions but with 


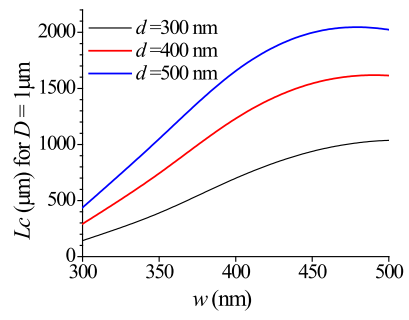

(a)

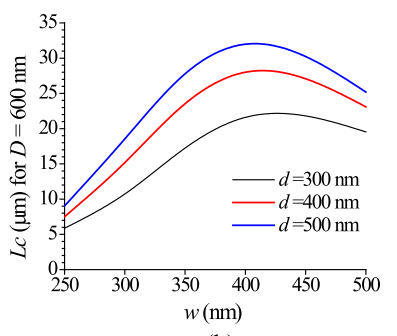

(b)
Fig. 7. Variation of the coupling length $\left(L_{c}\right)$ as a function of $w$ for different $d$ for silicon waveguide of square cross section. (a) $D=1 \mu \mathrm{m}$; (b) $D=600 \mathrm{~nm}$.

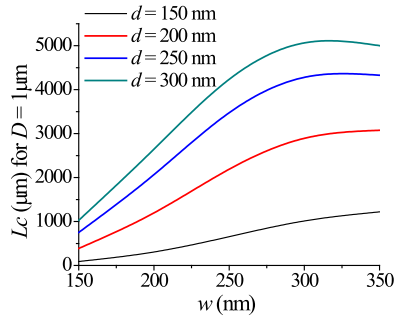

(a)

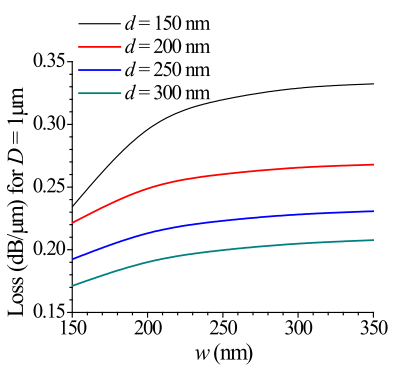

(b)

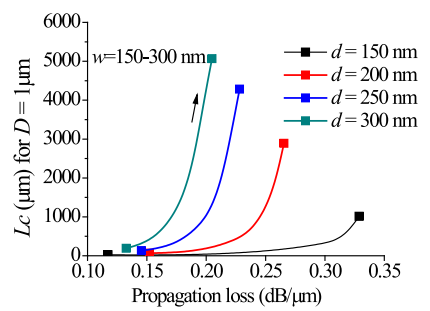

(c)

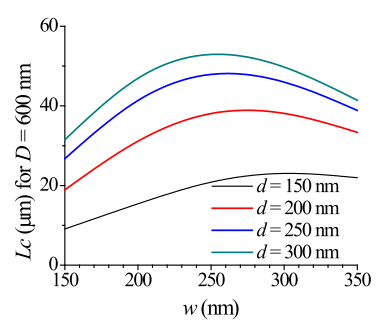

(d)

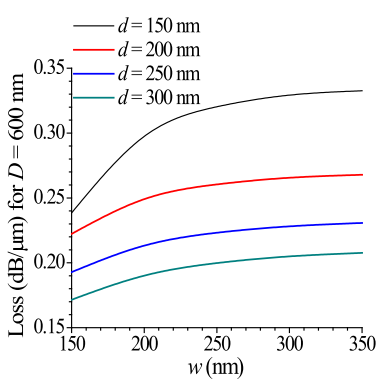

(e)

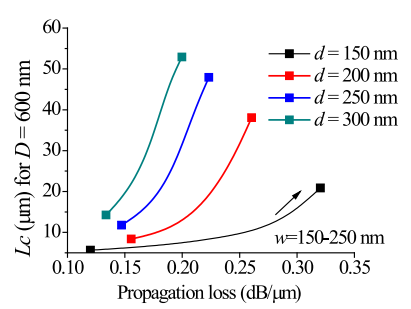

(f)
Fig. 8. Variation of the (a) coupling length $\left(L_{c}\right)$ and (b) propagation loss as a function of $w$ for different $d$, and (c) loss versus $L_{c}$ for the DLSPW with $D=1 \mu \mathrm{m}$. Variation of the (d) coupling length $\left(L_{c}\right)$ and (e) propagation loss as a function of $w$ for different $d$, and (f) loss versus $L_{c}$ for the DLSPW with $D=600 \mathrm{~nm}$.

a reduced waveguide spacing $(D=600 \mathrm{~nm})$. A reduction in the waveguide spacing drastically reduces the coupling length. It has been pointed out in [29], $L_{c}$ increases first and then decreases when $w$ increases for a fixed separation distance $D$. The reason is, when $w$ increases, the power is more confined to the waveguide core region; however, further increase $w$ results in a very small gap $(D-w)$ between the parallel waveguides, the coupling between the waveguides becomes stronger. For a waveguide that matches the single mode condition (for example, for $d=300 \mathrm{~nm}, w$ should be smaller than $400 \mathrm{~nm}$ ), the largest $L_{c}$ is about $500 \mu \mathrm{m}$ for $D=1 \mu \mathrm{m}$, and largest $L_{c}$ is less than $20 \mu \mathrm{m}$ for $D=600 \mathrm{~nm}$. For all the plasmonic waveguides analyzed later, we will also use $D=1 \mu \mathrm{m}$ and $D=600 \mathrm{~nm}$ to evaluate the integration density properties.

\section{B. Dielectric Loaded Surface Plasmon Waveguide}

Fig. 8 shows the coupling length $\left(L_{c}\right)$ and propagation loss of the DLSPW for a range of dimensions. As $d$ or $w$ increases, the core becomes larger and more power is confined in the core, which results in increase in $L_{c}$. However, for a fixed $d$, further increase in $w$ results in a smaller gap between the two waveguides, which results a decreasing $L_{c}$. At the same time, increasing $w$ leads the mode to extend more in lateral direction; thus the mode is affected more by the presence of the metal surface and the propagation loss increases. Comparison results for $D=1 \mu \mathrm{m}$ in Fig. 8(a) show a larger $L_{c}$ for a larger $w$ when $w$ is smaller than $300 \mathrm{~nm}$. Fig. 8(b) shows a larger $w$ associated with a larger propagation loss. Fig. 8(c) shows the relation between loss and $L_{c}$ for smaller waveguide width $(w=150-300 \mathrm{~nm})$ for $D=1 \mu \mathrm{m}$. Fig. 8(a)-(c) also shows a larger $d$ gives a better lateral confinement and less propagation loss. A similar trend is also observed for $D=600 \mathrm{~nm}$ (see Fig. 8(d) to (f)).

\section{Hybrid Plasmonic Waveguide}

In the HPWG a significant amount of light is confined in the low index spacer layer between the metal and the high index region. The level of light confinement depend on the dimensions $d, w$ and $g$. From our analysis we found that $d=300 \mathrm{~nm}$ provides good confinement at modest propagation loss, while also ensuring single mode operation. To keep the discussion brief, we do not present a detailed analysis of the effects of $d$ on the waveguide performance. Instead we present the results of our analysis on the effect of varying $w$ and $g$ for fixed $d(d=300$ $\mathrm{nm})$. Fig. 9 shows the relationship of $w$ with $L_{c}$ and propagation loss for different $g=10,20,50$, and $100 \mathrm{~nm}$. For a fixed value $w$, when $g$ increases, both $L_{c}$ and the propagation loss decrease because the mode is less confined in the gap and extends more into the silica cladding layer. At the same time, for the same $g$, increasing $w$ leads the mode to be confined in a larger core, which in turn increases $L_{c}$. Similar to other waveguides, for a very small $D(D=600 \mathrm{~nm})$ further increasing $w$ ( $w$ increased to $250-350 \mathrm{~nm}$ ) results in a much closer distance between the two waveguides, which results a decreasing in $L_{c}$.

\section{Plasmonic Slot Waveguide}

The plasmonic slot waveguide confines the mode inside the dielectric slot between two metal walls. Due to the very small penetration depth into the metal, most of the light coupling to the other waveguide is via the dielectric cladding. Such a waveguide with thicker metal (larger $d$ ) has a smaller cross talk (larger $L_{c}$ ). Similarly, the waveguide with a narrow gap (smaller g) also helps to confine more power inside the slot and results in a larger $L_{c}$. as shown in Fig. 10(a) and (d). Accordingly, for a fixed value $d$, when $g$ increases, the mode is less well confined in the gap and leaks into the cladding layer, and results in a lower loss as shown in Fig. 10(b) and (e). It has been shown 


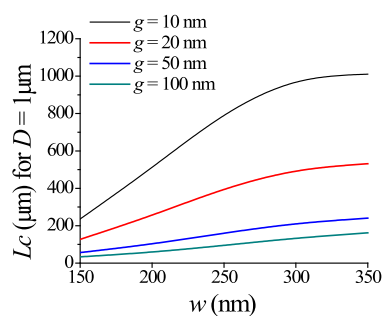

(a)

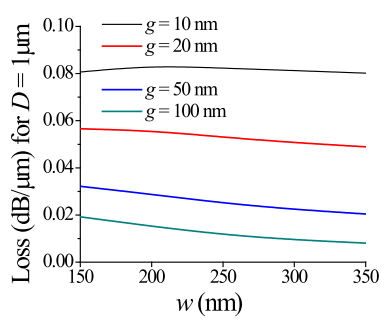

(b)

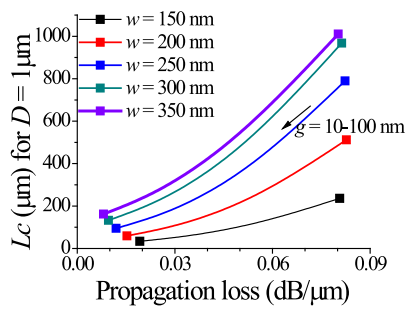

(c)

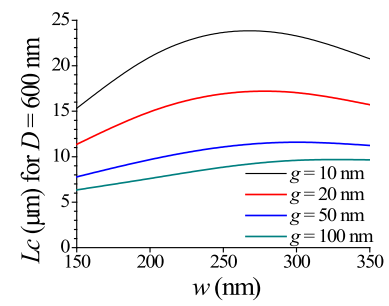

(d)

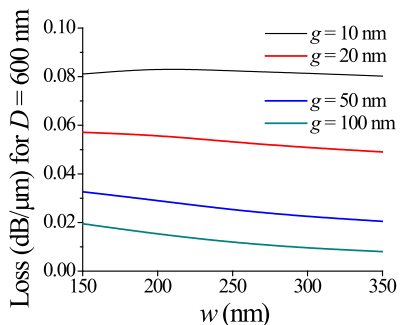

(e)

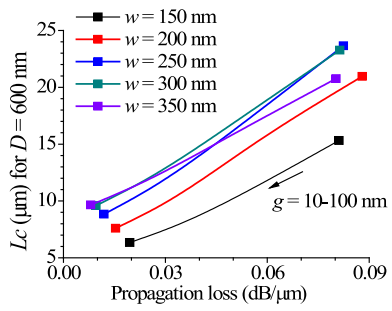

(f)
Fig. 9. Variation of the (a) Coupling length $\left(L_{c}\right)$ and (b) propagation loss as a function of $w$ for different $d$, and (c) loss versus $L_{c}$ for the HPWG with $D=1 \mu \mathrm{m}$. Variation of the (a) Coupling length $\left(L_{c}\right)$ and (b) propagation loss as a function of $w$ for different $d$, and (c) loss versus $L_{c}$ for the HPWG with $D=600 \mathrm{~nm}$.

in Fig. 10(c) and (f), $L_{c}$ is could be very large for the slot waveguide though with high loss.

\section{E. Hybrid Plasmonic Waveguide With a $90^{\circ}$ Rotation}

We have noticed the lateral confinement for the HPWG of Fig. 6(c) is comparable to the silicon waveguide in the previous sections. The reason is that the metal provides confinement in the vertical direction and does not greatly influence the confinement in the lateral direction. Here we consider a configuration where the HPWG structures are rotated by $90^{\circ}$ as shown schematically in Fig. 6(e); in this case the metal wall can enhance the lateral confinement, which is similar to the slot waveguide. We have optimized and fixed $w=200 \mathrm{~nm}$ to calculate the relationship of $g$ with $L_{c}$ and Propagation Loss for fixed $d=200,300$ and $400 \mathrm{~nm}$. One limit with this structure is that with a small $D$ and large $g$, the $90^{\circ}$ rotated HPWG (noted as HPWG_90 for short) starts supporting the hybrid modes between the two waveguides and this configuration is not very suitable. Therefore for $D=$ $600 \mathrm{~nm}, g$ should be smaller than $50 \mathrm{~nm}$.

The results of this analysis are shown in Fig. 11. It shows a similar trend to the plasmonic slot waveguide. For a fixed value $d$, when $g$ increases, both $L_{c}$ and Propagation Loss decreases, because the mode is less confined and leaks into the cladding layer.

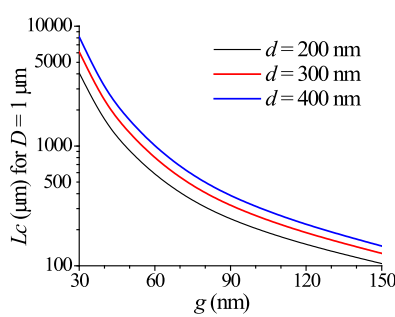

(a)

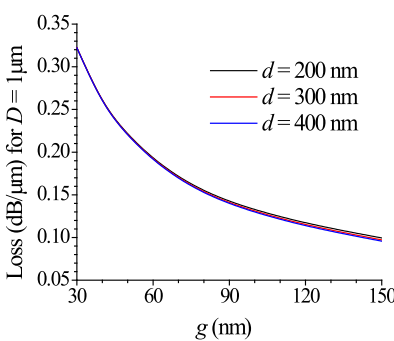

(b)

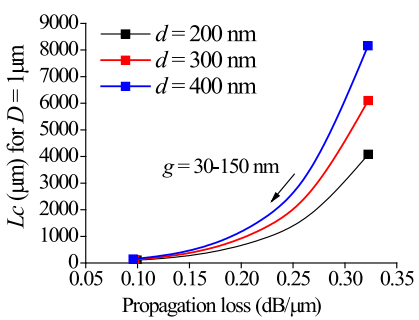

(c)

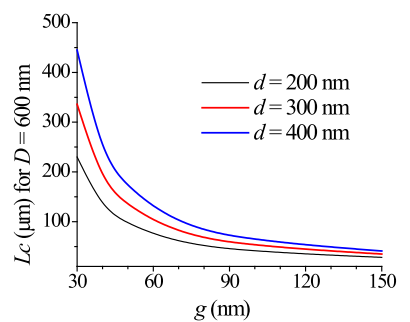

(d)

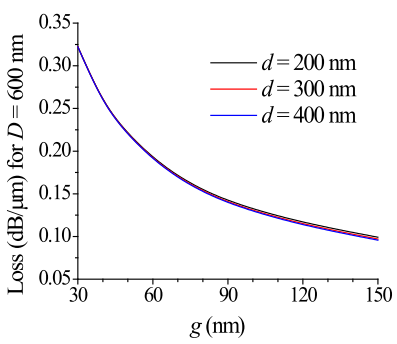

(e)

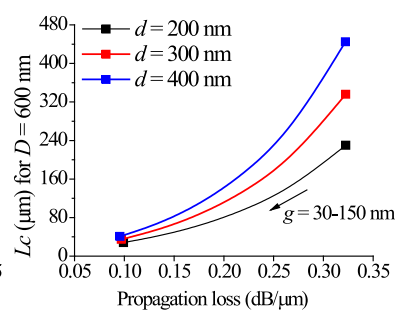

(f)
Fig. 10. Variation of the (a) coupling length $\left(L_{c}\right)$ and (b) propagation loss as a function of $g$ for different $d$, and (c) loss versus $L c$ for the slot waveguide with $D=1 \mu \mathrm{m}$. Variation of the (d) coupling length $\left(L_{c}\right)$ and (e) propagation loss as a function of $g$ for different $d$, and (f) loss versus $L c$ for the slot waveguide with $D=600 \mathrm{~nm}$.

\section{DISCUSSIONS}

In the previous two sections we have analyzed the characteristics of silicon waveguide and various plasmonic waveguides in detail. Here we summarize the results of these results and compare the performance of various waveguides.

\section{A. Comparison of Power Density and Confinement of Various Plasmonic Waveguides}

In Section II we have presented a detailed discussion of power confinement and guided power density of various waveguides for various choices of waveguide dimensions. As we have seen from that discussion, for all plasmonic waveguides, an increased power density is always accompanied by an increased propagation loss. To determine which waveguide offers the best tradeoff between loss and power density, we have plotted the power density achievable for the different plasmonic waveguides as a function of the corresponding propagation loss in Fig. 12(a). For clarity we choose one typical case for each waveguide. The arrows in Fig. 12 indicate increasing dimensions $(d$ or $g$ ). For the DLSPW the width ( $w$ ) is fixed at $200 \mathrm{~nm}$ and the height of the silicon layer $(d)$ is varied from 150 to $300 \mathrm{~nm}$. The corresponding $D$ for the DLSPW varies from 13 to $24 \mathrm{mw} / \mu \mathrm{m}^{2}$. For 


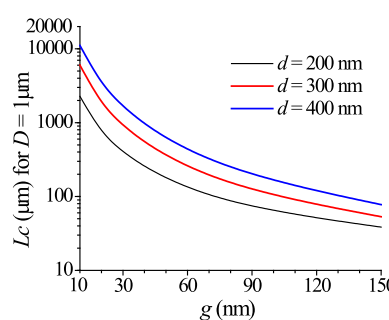

(a)

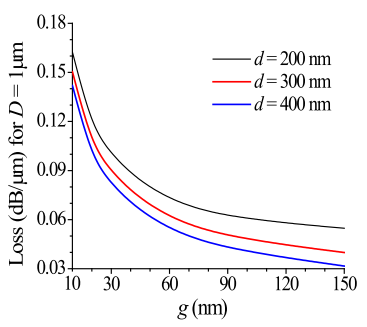

(b)

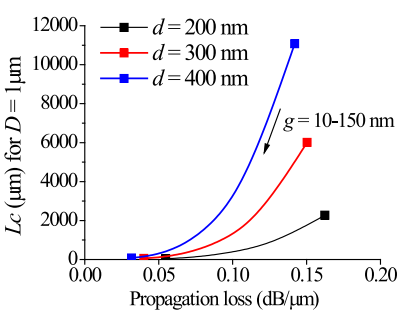

(c)

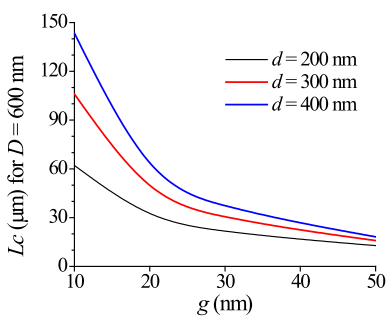

(d)

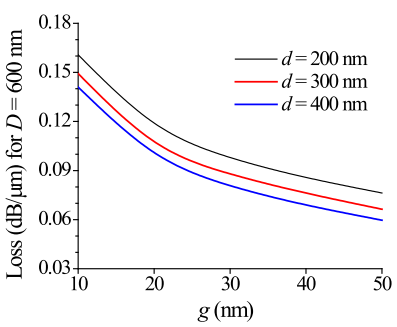

(e)

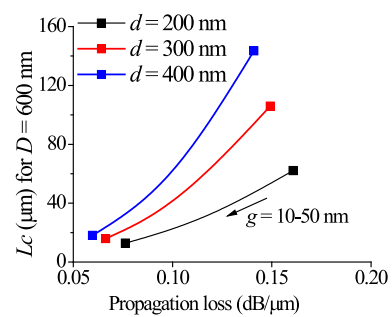

(f)
Fig. 11. Variation of the (a) coupling length $\left(L_{c}\right)$ and (b) propagation loss as a function of $g$ for different $d$, and (c) loss versus $L c$ for the HPWG_90 with $D=1 \mu \mathrm{m}$. Variation of the (a) coupling length $\left(L_{c}\right)$ and (b) propagation loss as a function of $g$ for different $d$, and (c) loss versus $L c$ for the HPWG_90 with $D=600 \mathrm{~nm}$.

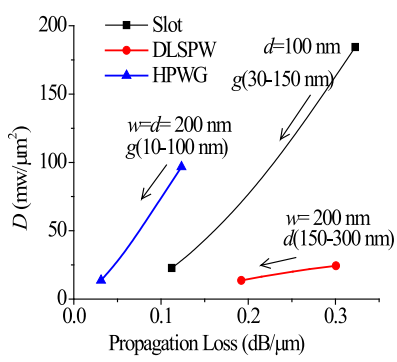

(a)

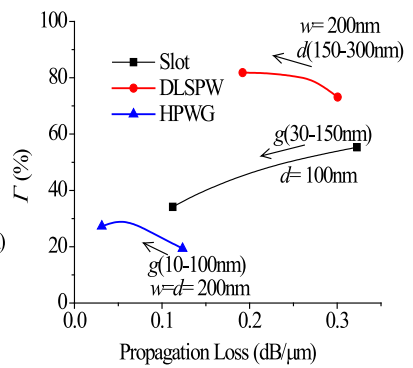

(b)
Fig. 12. (a) Normalized power density versus propagation loss for three types of plasmonic waveguides. (b) Confinement factor versus propagation loss for three types of plasmonic waveguides.

the slot waveguide, the metal thickness $(d)$ is fixed at $100 \mathrm{~nm}$ and the slot width $(g)$ is varied from 30 to $100 \mathrm{~nm}$. This guide can provide the highest power density which can be approximately $200 / \mu \mathrm{m}^{2}$. For the HPWG, both the width $(w)$ and silicon thickness $(d)$ have been fixed at $200 \mathrm{~nm}$ and the gap $(g)$ is varied from 10 to $100 \mathrm{~nm}$. The power density achievable for the HPWG is higher than the DLSPW but lower compared to the slot waveguide. However, for a given power density the propagation loss of the HPWG is much lower compared to the other types of plasmonic guides.

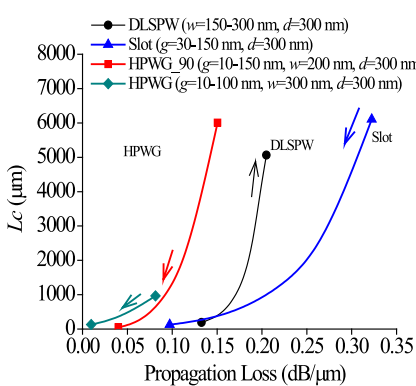

(a)

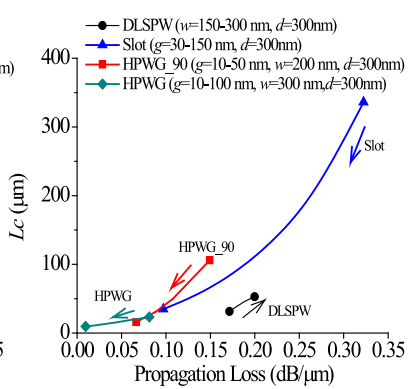

(b)
Fig. 13. (a) Coupling length $\left(L_{c}\right)$ versus propagation loss with $D=1 \mu \mathrm{m}$ for the DLSPW, the Plasmonic slot waveguide, the HPWG, and the HPWG_90. (b) Coupling length $\left(L_{c}\right)$ versus propagation loss with $D=600 \mathrm{~nm}$ for the DLSPW, the Plasmonic slot waveguide, the HPWG, and the HPWG_90.

Plasmonic waveguides also exhibit a tradeoff between confinement and propagation loss. Fig. 12(b) shows the confinement factor achievable for the plasmonic guides under consideration. The dimensions chosen are same as those in Fig. 12(a). The confinement is best for the DLSPW (85\%) and comparable to that achievable from silicon nanowire waveguide. The confinement is relatively low for the HPWG (up to $30 \%$ ) compared to the other guides but its propagation loss is also much lower.

From the analysis above we observe that the confinement of the DLSPW is very similar to the silicon waveguide, but it suffers from a very high propagation loss. Therefore, DLSPW may not be a suitable choice for applications where a high power density is required, for example in nonlinear optics. The slot waveguide can achieve very high power density and good confinement but the propagation loss is also very high. HPWG is a compromise in the sense that it has significantly lower loss but still can provide high power density and moderate confinement. The choice between HPWG and slot waveguides should depend on the relative importance of power density and power confinement for a specific application.

\section{B. Comparison of Coupling Lengths of Various Plasmonic Guides}

Fig. 13(a) and (b) show coupling length and propagation loss achievable for various waveguides for waveguide spacing $(D)$ of $1 \mu \mathrm{m}$ and $600 \mathrm{~nm}$, respectively. For clarity we choose one typical case for each waveguide. Similar to the figures of Section V-A, the arrows in Fig. 13 indicate increasing dimensions ( $g$ or $w$ ). As Fig. 13(a) indicates, for $D=1 \mu \mathrm{m}$, for a given coupling length, the $90^{\circ}$ rotated HPWG provides the best compromise between crosstalk and propagation loss. In Fig. 13(b) The HPWG exhibits low loss like the previous case (Fig. 13(a)) but in this case the coupling length is much less than the plasmonic slot waveguide. The slot waveguide can achieve a very high density of integration, though at the cost of a very large propagation loss. Thus for a highly dense packaging, the slot waveguide shows its advantages over others.

\section{CONCLUSION}

We have analyzed and compared the performance of three popular plasmonic waveguides in terms of power density, 
coupling lengths and propagation loss. Several conclusions can be reached from this analysis, especially about the applications of these waveguides in high power intensity and high packing density optical devices.

We found that DLSPW can confine a large amount of the power inside silicon region, but the corresponding power density is low. Therefore it may not be a very attractive choice for applications need high power intensity like nonlinear applications. The slot waveguide can achieve very high power density with larger loss. The power density achievable for the HPWG is higher than the DLSPW, but is lower than the slot waveguide; however, for a given power density level, the propagation loss of the HPWG is much smaller.

Regarding the achievable density of integration, the plasmonic slot waveguides can achieve very high packing density, which is not possible by other types of plasmonic waveguides but this large packing density comes at the cost of very large propagation loss. HPWG offers longer coupling length for a lower propagation loss. For the same propagation loss the packing density achievable is better than that for the case of slot and DLSPW unless the waveguide spacing is too small.

\section{REFERENCES}

[1] E. Ozbay, "Plasmonics: Merging photonics and electronics at nanoscale dimensions," Science, vol. 311, no. 5758, pp. 189-193, Jan. 2006.

[2] W. L. Barnes, A. Dereux, and T. W. Ebbesen, "Surface plasmon subwavelength optics," Nature, vol. 424, pp. 824-830, 2003.

[3] E. Feigenbaum and M. Orenstein, "Modeling of complementary plasmon waveguiding," J. Lightw. Technol., vol. 25, no. 9, pp. 2547-2562, Sep. 2007.

[4] A. V. Krasavin and A. V. Zayats, "Silicon-based plasmonic waveguides," Opt. Exp., vol. 18, pp. 11791-11799, 2010.

[5] G. Veronis and S. Fan, "Guided subwavelength plasmonic mode supported by a slot in a thin metal film," Opt. Lett., vol. 30, pp. 3359-3361, 2005.

[6] M. Z. Alam, J. S. Aitchison, and M. Mojahedi, "A marriage of convenience: Hybridization of surface plasmon and dielectric waveguide modes," Laser Photon. Rev., vol. 8, no. 3, pp. 394-408, 2014.

[7] M. Z. Alam, J. Meier, J. S. Aitchison, and M. Mojahedi, "Propagation characteristics of hybrid modes supported by metal-low-high index waveguides and bends," Opt. Exp., vol. 18, pp. 12971-12979, 2010.

[8] X. Sun, M. Alam, S. Wagner, J. Aitchison, and M. Mojahedi, "Experimental demonstration of a hybrid plasmonic transverse electric pass polarizer for a silicon-on-insulator platform," Opt. Lett., vol. 37, pp. 4814-4816, 2012.

[9] F. Lu et al., "Surface plasmon polariton enhanced by optical parametric amplification in nonlinear hybrid waveguide," Opt. Exp., vol. 19, pp. 2858 2865, 2011.

[10] A. R. Davoyan, I. V. Shadrivov, A. A. Zharov, D. K. Gramotnev, and Y.S. Kivshar, "Nonlinear nanofocusing in tapered plasmonic waveguides,' Phys. Rev. Lett., vol. 105, art. no. 116804, pp. 1-4, 2010.

[11] J. Homola, S. S. Yee, and G. Gauglitz, "Surface plasmon resonance sensors: Review," Sens. Actuators B, Chem., vol. 54, no. 1, pp. 3-15, Jan 1999.

[12] F. Bahrami, M. Maisonneuve, M. Meunier, J. S. Aitchison, and M. Mojahedi, "An improved refractive index sensor based on genetic optimization of plasmon waveguide resonance," Opt. Exp., vol. 21, no. 18, pp. 20863-20872, 2013.

[13] M. Z. Alam, F. Bahrami, J. S. Aitchison, and M. Mojahedi, "Analysis and optimization of hybrid plasmonic waveguide as a platform for biosensing," IEEE Photon. J.,, vol. 6, no. 4, pp. 1-10, Aug. 2014.

[14] I. Rukhlenko, M. Premaratne, and G. Agrawal, "Effective mode area and its optimization in silicon-nanocrystal waveguides," Opt. Lett., vol. 37, pp. 2295-2297, 2012.

[15] I. D. Rukhlenko, M. Premaratne, and G. P. Agrawal, "Nonlinear silicon photonics: analytical tools," IEEE J. Sel. Top. Quantum Electron., vol. 16, no. 1, pp. 200-215, Jan./Feb. 2010
[16] F. Grillot, L. Vivien, S. Laval, and E. Cassan, "Propagation loss in singlemode ultrasmall square silicon-on-insulator optical waveguides," Lightw. Technol., J. , vol. 24, no. 2, pp. 891-896, Feb. 2006.

[17] R. Buckley and P. Berini, "Figures of merit for 2D surface plasmon waveguides and application to metal stripes," Opt. Exp., vol. 15, pp. 12174 12182, 2007.

[18] R. F. Oulton, G. Bartal, D. F. P. Pile, and X. Zhang, "Confinement and propagation characterisics of subwavelength plasmonic modes," New J. Phys., vol. 10, art. no. 105018, pp. 1-14, 2008.

[19] T. D. Visser, H. Blok, B. Demeulenaere, and D. Lenstra, "Confinement factors and gain in optical amplifiers," IEEE J. Quantum Electron., vol. 33, no. 10 , pp. $1763-1766$, Oct. 1997

[20] R. Zia, M. D. Selker, P. B. Catrysse, and M. L. Brongersma, "Geometries and materials for subwavelength surface plasmon modes," J. Opt. Soc. Amer. A, vol. 21, pp. 2442-2446, 2006.

[21] A. A. Maradudin, J. R. Sambles, and W. L. Barnes, "Waveguiding with surface plasmon polaritons," in Mondern Plasmonics, 1st ed. Amsterdam, The Netherlands: Elsevier, 2014, pp. 171-172.

[22] A. Yariv, "Coupled-mode theory for guided-wave optics," IEEE J. Quantum Electron., vol. 9, no. 9, pp. 919-933, Sep. 1973.

[23] A Noual, A. Akjouj, Y. Pennec, J-N. Gillet, and B. Djafari-Rouhani, "Modeling of two-dimensional nanoscale Y-bent plasmonic waveguides with cavities for demultiplexing of the telecommunication wavelengths," New J. Phys., vol. 11, art. no. 103020, pp. 1-19, 2009.

[24] E. P. Li and H. S. Chu, "Passive plasmonicwaveguide-based devices," in Plasmonic Nanoelectronics and Sensing, 1st ed. Cambridge, U.K.: Cambridge Univ. Press, 2014.

[25] P. B. Johnson and R. W. Christy, "Optical constants of the noble metals," Phys. Rev. B, vol. 6, pp. 4370-4379, 1972.

[26] E. D. Palik, Handbook of Optical Constants of Solids. Orlando, FL, USA Academic, 1985

[27] V. Almeida, Q. Xu, C. Barrios, and M. Lipson, "Guiding and confining light in void nanostructure," Opt. Lett., vol. 29, pp. 1209-1211, 2004.

[28] G. Veronis and S. Fan, "Crosstalk between three-dimensional plasmonic slot waveguides," Opt. Exp., vol. 16, pp. 2129-2140, 2008.

[29] D. Dai, Y. Shi, and S. He, "Characteristic analysis of nanosilicon rectangular waveguides for planar light-wave circuits of high integration," Appl. Opt., vol. 45, no. 20, pp. 4941-4946, 2006.

X. Sun (S'11) received the B.Sc. degree in engineering from the University of Electronic Science and Technology, Chengdu, China, in 1999, and the MA.Sc. degree from the University of Toronto, Toronto, Canada, in 2013.

She is currently working toward the $\mathrm{Ph} . \mathrm{D}$. degree in the Photonics Group, the University of Toronto. Her research interests include plasmonics and integrated optics.

M. Z. Alam (S'10-M'13) received the MA.Sc. degree from the University of Victoria, Victoria, AB, Canada, in 2004, and the Ph.D. degree from the University of Toronto, Toronto, ON, Canada, in 2012. For his Ph.D. research work, he received the Douglas R. Colton Medal for Research Excellence.

He is currently a Postdoctoral Fellow in the Photonics Group, the University of Toronto. His research interests include plasmonics, integrated optics, electromagnetics, biosensing, and optical gas sensing.

M. Mojahedi (S'97-M'98-SM'05) received the Ph.D. degree from the Center for High Technology Materials, University of New Mexico, Albequerque, USA, in December 1999.

$\mathrm{He}$ is currently a Professor in the Photonics Group, University of Toronto. His research interests include matter wave interactions, plasmonics, integrated optics, metamaterials, dispersion engineering, quantum dots, and wells lasers, fundamental electromagnetic theory.

J. S. Aitchison (M'96-SM'00) received the B.Sc. (First Class Hons.) and the Ph.D. degrees from Heriot-Watt University, Edinburgh, U.K., in 1984 and 1987, respectively.

He is currently a Professor in the Photonics Group, University of Toronto, Toronto, ON, Canada. His current research interests include all-optical switching and signal processing, plasmonics, optoelectronic integration, and optical biosensors. 